\title{
Deficits in functional performance and gait one year after total knee arthroplasty despite improved self-reported function
}

\author{
Josefine E. Naili ${ }^{1}$ Maura D. Iversen ${ }^{1,2,3}$ • Anna-Clara Esbjörnsson ${ }^{1}$ • \\ Margareta Hedström ${ }^{4,5}$ • Michael H. Schwartz ${ }^{6,7,8}$ • Charlotte K. Häger9 • \\ Eva W. Broström ${ }^{1}$
}

Received: 30 March 2016 / Accepted: 5 July 2016 / Published online: 19 July 2016

(C) The Author(s) 2016. This article is published with open access at Springerlink.com

\begin{abstract}
Purpose The current literature lacks sufficient information about improvements in gait patterns and function after total knee arthroplasty (TKA) and whether patients return to full function. This study evaluated change in gait, performancebased function, and self-reported function 1 year after TKA in patients with symptomatic knee osteoarthritis and how these aspects interrelate.

Methods A total of 28 patients (64\% female) with knee osteoarthritis, with a mean age of $66( \pm 7)$ years, and 25 age- and gender-matched controls participated in this prospective cohort study. Three-dimensional gait analysis
\end{abstract}

Josefine E. Naili

josefine.naili@ki.se

Maura D. Iversen

m.iversen@neu.edu

Anna-Clara Esbjörnsson

anna-clara.esbjornsson@ki.se

Margareta Hedström

margareta.hedstrom@karolinska.se

Michael H. Schwartz

schwa021@umn.edu

Charlotte K. Häger

charlotte.hager@umu.se

Eva W. Broström

eva.brostrom@ki.se

1 Department of Women's and Children's Health, Karolinska Institutet, Karolinska University Hospital, Stockholm, Sweden

2 Department of Physical Therapy, Movement \& Rehabilitation Sciences, Bouve College of Health Sciences, Northeastern University, Boston, MA, USA generated comprehensive measures of kinematic and kinetic gait deviations, respectively. Participants completed the Five Times Sit-to-Stand (5STS) test, and the self-reported questionnaire Knee Injury and Osteoarthritis Outcome Score (KOOS), at baseline prior to surgery and 1 year after TKA. Results Kinetic gait deviations of both the operated and non-operated limb persisted in patients with knee osteoarthritis at 1 year after surgery, while kinematic gait patterns were comparable to controls. Performance on the 5STS and KOOS scores in patients with knee osteoarthritis improved significantly 1 year after surgery (effect size $0.5-1.5$ ), but did not reach the level of controls. Ten patients with knee 
osteoarthritis (36\%) exceeded the minimally detectable change on the 5STS.

Conclusion Measures of overall gait patterns and the 5STS revealed improvements in function 1 year after TKA, but were not restored to the level of healthy controls. Based on change in 5STS performance, we identified patients with substantial improvements in gait patterns. Self-reported measures of function could not detect differences between patients improving in 5STS performance and those who did not. These findings highlight the use of the 5STS in clinical practice since improvement on this test seems to follow the reduction in gait pattern deviations.

Level of evidence II.

Keywords Arthroplasty · Knee osteoarthritis · Outcome · Gait · Function - Biomechanics · Patient-reported outcome · Mobile bearing

\section{Introduction}

The current literature lacks sufficient information about the degree of functional improvement after total knee arthroplasty (TKA) using objectively measured function including gait patterns [21]. Patients with knee osteoarthritis report decreased pain and improved function following TKA surgery $[15,19]$, and research indicates patient satisfaction correlates well with both self-reported outcomes and a clinicianbased score [2]. Mean improvement in self-reported pain and function is reported to range from 50 to $168 \%$ at 6 months after TKA [35]. At 1 year after TKA, improvements in selfreported pain and function range from 52 to $194 \%$, where the largest improvements were found in a functional subscale, measuring function in sport and recreation [33]. Selfreported measures of function have been found to be largely influenced by pain, and if perceived pain is greatly reduced, the assessment of function may be overestimated or confused with improved function rather than reduced pain [3].

Despite these improvements, $20-30 \%$ of patients report persistent disability, limited function, reduced quality of life, diminished working capacity, and gait deviations after TKA $[24,38]$. Gait pattern in patients operated with unicompartmental knee arthroplasty resembles healthy controls more than patients operated with TKA [37]. In patients with knee osteoarthritis, joint replacements are generally considered successful surgeries. Traditionally, these surgeries were performed on older patients with lower functional demands. Thus, functional improvement has not been considered as important as pain relief. Disability associated with TKA may be conceptualized as a surgical failure since the indication for surgery is pain and impaired function. In clinical practice, the array of available functional outcome measures is usually restricted to self-reported measures and performance-based measures as they do not require special equipment. Detailed knowledge of the relationships between objective measures of function derived from threedimensional (3D) gait analysis and performance-based function would be advantageous when determining which outcome measures to use in clinical practice.

This study aimed to objectively quantify functional improvements in gait patterns and performance-based function 1 year after TKA in patients with knee osteoarthritis. Further, we aimed to explore the relationships between the degree of improvement in gait pattern, with respect to kinematics and kinetics, in performance-based function using the Five Times Sit-to-Stand (5STS) test, and in self-reported function using a disease-specific questionnaire. In this study, it was hypothesized that (1) patients with knee osteoarthritis would significantly reduce the degree of gait pattern deviation and (2) improve performance on the 5STS 1 year after TKA. Further it was hypothesized that patients with knee osteoarthritis, operated with TKA, would nevertheless not reach the level of healthy controls with regard to gait patterns and performance on the 5STS 1 year after surgery.

\section{Materials and methods}

Forty patients with physician diagnosed primary knee osteoarthritis were included in this prospective cohort study. Patients were recruited from two orthopaedic departments in Stockholm, Sweden (Ortho center, Löwenströmska hospital and Karolinska University Hospital). The inclusion criteria were: being scheduled for TKA within one month; ability to walk $10 \mathrm{~m}$ repeatedly without the use of a walking aid; and ability to understand verbal and written information in Swedish. Exclusion criteria were: any previous major orthopaedic surgery in the lower limbs, severe back pain or other lower extremity joint pain, rheumatoid arthritis, diabetes mellitus, neurological disease, BMI $>40$, and/ or other condition affecting walking ability. Twenty-five age- and gender-matched, healthy controls without any known musculoskeletal disease or neurological disorder were recruited through acquaintances between the years 2013 and 2015. The control group was matched to the osteoarthritis group by age strata across five age groups (40-49, 50-59, 60-69, 70-79, and 80-89 years of age). The regional ethical review board in Stockholm, Sweden, approved the study (DNR 2010/1014-31/1). All participants provided informed consent in accordance with the Declaration of Helsinki.

Twenty-eight patients with knee osteoarthritis, with a mean age of $66( \pm 7)$ years, $64 \%$ female, completed the 1-year follow-up (Table 1). The most common reasons for not completing the 1-year follow-up were TKA in the contralateral limb within the subsequent year $(n=5)$, not 
Table 1 Baseline characteristics and clinical features of patients with knee osteoarthritis scheduled for knee arthroplasty and in the control group

\begin{tabular}{|c|c|c|c|}
\hline & Knee OA $(n=28)$ & $\begin{array}{l}\text { Control group } \\
(n=25)\end{array}$ & $\begin{array}{l}\text { Differences } \\
\text { between groups }\end{array}$ \\
\hline Characteristics & & & $p$ value \\
\hline Mean age, years (SD) & $65.7(7.3)$ & $65.7(9.5)$ & n.s. \\
\hline $40-49$ years, $n(\%)$ & 0 & $1(4)$ & \\
\hline $50-59$ years, $n(\%)$ & $7(25)$ & $5(20)$ & \\
\hline $60-69$ years, $n(\%)$ & $12(43)$ & $9(36)$ & \\
\hline $70-79$ years, $n(\%)$ & $8(29)$ & $9(36)$ & \\
\hline $80-89$ years, $n(\%)$ & $1(4)$ & $1(4)$ & \\
\hline Female, $n(\%)$ & $18(64)$ & $16(64)$ & n.s. \\
\hline Body mass index $\left(\mathrm{kg} / \mathrm{m}^{2}\right)$, mean $(\mathrm{SD})$ & $29.6(4.6)$ & $24.9(2.9)$ & 0.003 \\
\hline Body weight (kg), mean (SD) & $83.7(12.8)$ & $72.8(12.2)$ & 0.000 \\
\hline Height $(\mathrm{cm})$, mean $(\mathrm{SD})$ & $168(8)$ & $171(8)$ & n.s. \\
\hline Symptom duration (years), mean (SD) & $7.8(7.7)$ & NA & \\
\hline Previous minor orthopaedic surgery, $n(\%)$ & $14(50)$ & NA & \\
\hline \multicolumn{4}{|l|}{ Modified KL score $(1-4 b)$} \\
\hline $1-2$ & - & NA & \\
\hline $3 \mathrm{a}, n(\%)$ & $1(4)$ & NA & \\
\hline $3 \mathrm{~b}, n(\%)$ & $4(14)$ & NA & \\
\hline $4 \mathrm{a}, n(\%)$ & $7(25)$ & NA & \\
\hline $4 \mathrm{~b}, n(\%)$ & $16(57)$ & NA & \\
\hline \multicolumn{4}{|l|}{ Use of analgesics } \\
\hline Daily use, $n(\%)$ & $9(32)$ & NA & \\
\hline If necessary (when needed), $n(\%)$ & $11(39)$ & NA & \\
\hline Never (rarely), $n(\%)$ & $8(29)$ & NA & \\
\hline
\end{tabular}

$O A$ osteoarthritis; $y$ years; $S D$ standard deviation; $n$ number; $K L$ Kellgren and Lawrence; $E Q-5 D$ EuroQol Five Dimensions; minor orthopaedic surgery refers to knee joint arthroscopy for all but two in the knee OA group which refers to surgical treatment of hallux valgus. Parametric statistics, independent samples $t$ tests, were used to calculate differences between OA group and controls. Level of significance set to $p<0.05$

undergoing the planned surgery $(n=2)$, or post-operative infection causing re-operation $(n=2)$ (Fig. 1). Patients with knee osteoarthritis who did not complete the 1-year follow-up $(n=12)$ did not differ statistically from the studied TKA group with respect to the distribution of age, gender, weight, height, BMI, or duration of years with symptomatic knee osteoarthritis (which was evaluated using independent samples $t$ tests or the Mann Whitney $U$ test, depending on the distribution of data, and a Fishers exact test).

\section{Protocol}

Gait analyses were conducted, and performance-based function was assessed by one of the two experienced physiotherapist (JEN, ACE) at the Motion Analysis Laboratory at Karolinska University Hospital, between the years 2010 and 2015. Each test session started with a physical examination using a standardized protocol. Anthropometric measures were recorded using calibrated scales. Participants started with completing the gait trials, walking barefoot along a 10-m-long pathway at self-selected speed. Recordings were performed in two directions (back and forth). Walking speed, kinematic, and kinetic parameters were collected using an 8-camera motion system (@Vicon Motion Systems Ltd, Oxford, UK) and two force plates (Kistler, Winterthur, Switzerland). Kinetics were expressed as internal moments and total joint power. We used the biomechanical model Plug-In-Gait, where 35 retro-reflective markers were placed on anatomical landmarks [8]. Using this model, good intra-sessional repeatability has been reported [13] and an inter-sessional standard error of $1.8^{\circ}$ for global kinematic data in healthy adults [12].

After completing the gait trials, participants completed self-reported measures to allow for a rest period $(\sim 20 \mathrm{~min})$, before performing the 5STS [22]. Patients with knee osteoarthritis performed the test protocol twice: once within one month prior to TKA and once again 1 year after surgery. The post-operative evaluation occurred at a median of 12 months (range 11-14 months) following TKA. At the post-operative assessment, patients provided information 
Fig. 1 Flowchart of included patients with knee osteoarthritis (OA), test procedures, excluded patients, and patients completing the 1-year follow-up

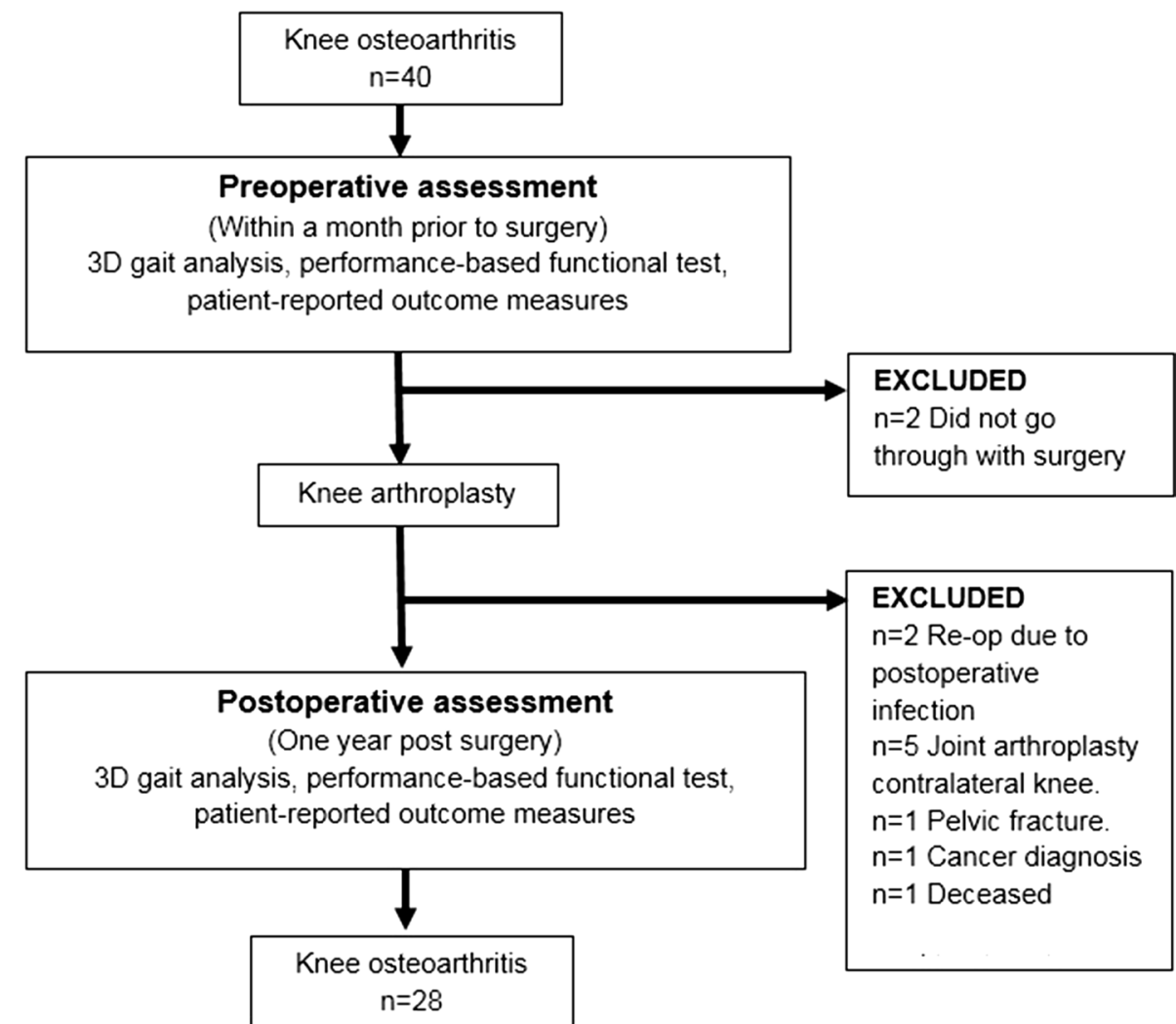

on their post-operative rehabilitation, duration of rehabilitation, and post-operative complications (e.g. fall accidents, deep vein thrombosis, and wound infections).

\section{Knee replacement surgery and post-operative rehabilitation}

Seven senior orthopaedic surgeons from two different hospitals performed the surgeries. All procedures were performed using a posterior cruciate ligament-retaining cemented TKA.

Post-operative regimes allowed full weight-bearing (together with use of an appropriate walking aid), and rehabilitation was performed according to standard practice at each hospital. Thereafter, rehabilitation was provided in a primary care setting of the patients' choice. The standard post-operative rehabilitation lasted for a median duration of 3 months (range 1-6 months) after TKA.

\section{Measures of self-reported function and pain}

All study participants completed the Knee Injury and Osteoarthritis Outcome Score (KOOS) [29], which is considered a reliable measure of baseline function and change over time in patients with knee osteoarthritis [7, 34]. The KOOS is divided into five subscales: symptoms, pain, function in activities of daily living (ADL), function in sport and recreation, and knee-related quality of life. All subscales demonstrate adequate test-retest reliability (intra class correlation (ICC) range 0.85-0.9) [7]. Each subscale generates a final score ranging from 0 to 100 where 0 represents "worst" and 100 "best" [29].

Participants also completed a health-related quality of life (HRQoL) questionnaire, the EuroQol five dimensions (EQ-5D) [4], which is considered valid and responsive in patients with chronic pain, including knee osteoarthritis [28]. In the present study, we used the EQ-5D version with three response options for each item (EQ-5D-3L), and the UK EQ-5D value set to obtain a single index value for health state [10].

\section{Measures of radiographic severity}

Pre-operative radiographs were collected according to standard procedures at each hospital. Two experienced orthopaedic surgeons (MH, PG) assessed the radiographs together and provided the radiologic classification of osteoarthritis, according to the modified Kellgren and Lawrence's classification (KL) ranging from grades I to IV [9]. Radiographs defined as KL scores of 3 to 4 were further subclassified, by incorporating scores of joint space narrowing (JSN) and bone attrition. Thus, a KL grade 3 radiograph with mild JSN was graded 3 a and radiographs with more severe JSN 3b. A KL grade 4 radiograph 
demonstrating complete loss of joint space was divided into 4a if there was no bone attrition and $4 \mathrm{~b}$ if subchondral bone attrition existed [9].

\section{Objective measures of gait patterns and function}

Three-dimensional gait analyses were performed, rendering measures of overall gait pattern deviations, using the Gait Deviation Index for kinematics (GDI) and kinetics (GDI-kinetic). The GDI and GDI-kinetic allow comparison of an individual's gait pattern against the gait of a reference group ( $n=59$ for GDI, $n=56$ for GDI-kinetic) [11, 30, 32]. Reference subjects were selected from the control database at the Motion Analysis Laboratory. The GDI is calculated from the pelvis and hip kinematics in all three anatomical planes, the knee and ankle in the sagittal plane and foot progression in the transversal plane [32]. The GDI-kinetic is calculated from the hip, knee, and ankle moments in the frontal and sagittal plane and total joint power in the hip, knee, and ankle [30]. Each limb is considered independently. A GDI or GDI-kinetic score of $\geq 100$ represents normal gait pattern, whereas each ten-point decrement below 100 represents one standard deviation from normal gait and indicates deviation in kinematics (GDI) or kinetics (GDI-kinetic) in one or more joints. In patients with rheumatoid arthritis, ICC of the GDI is 0.952 and standard error of measurement (SEM) 1.92 scores [11]. Five gait trials, with clean force plate strikes, were analysed for each participant, at each test session (pre- and post-operative). The GDI and GDI-kinetic were averaged for these trials to obtain one value for each limb (operated, non-operated) for each index. Walking speed was normalized by gravity and leg length, as described by Hof [16]. Calculations of 3D gait analysis data were performed using the software program MATLAB ${ }^{\circledR}$, R2014a (The MathWorks, Inc, Natick, MA).

The Five Times Sit-to-Stand (5STS) test is a timed test where the participant is asked to rise from a seated position to a standing position five times as quickly as possible [22]. Performance on this test is associated with quadriceps strength and functional performance up to 6 months after TKA in patients with knee osteoarthritis [6]. The test shows excellent relative and absolute reliability in older adults (ICC 0.95, SEM $0.9 \mathrm{~s}$ ) [14]. The test was performed twice, timed to a hundredth of a second, and the best (lowest) value was used in the analysis. To determine post-operative improvement in objectively measured function, the 5STS was used as it is a method easily applicable in clinical practice. Patients with knee osteoarthritis were grouped and compared based on their 5STS results according to the established minimally detectable change (MDC) of $2.5 \mathrm{~s}$ in 5STS performance [14]. Patients with a reduction in time equal to or greater than $2.5 \mathrm{~s}$ were considered to have a
"Good 5STS outcome", and those with a reduction of less than $2.5 \mathrm{~s}$ or an increase in time were considered to have a "Bad 5STS outcome".

\section{Statistical analysis}

Statistical analyses were performed using IBM SPSS Statistics version 22. A significance level was set at $p<0.05$. To assess change in function pre- and post-operatively, paired sample $t$ tests or Wilcoxon signed-rank tests were used, depending on the distribution of data. Normal distribution of the data was assessed using Shapiro-Wilk's test and Q-Q plots. To evaluate the magnitude of change in function, effect sizes (Cohen's $d$ ) were calculated along with $95 \%$ confidence intervals (CI) [26]. A Fishers exact test was used to determine whether the proportion of improvement on the 5STS was beyond the MDC, and change in GDI and GDIkinetic was statistically different [22]. Groups were defined according to the MDC of each specific measurement, change in time by $2.5 \mathrm{~s}$ on the 5STS [14] and change by 5.4 units in GDI and GDI-kinetic scores, respectively [11].

To evaluate differences in function between patients with TKA and controls, independent samples $t$ tests or the Mann Whitney $U$ test was used, depending on data distribution. To determine the sample size needed to detect a difference of 5 GDI units between patients with knee osteoarthritis and healthy controls, and with the power set at 0.8 , a sample size of 24 subjects was required in the knee osteoarthritis group. Since the control group consisted of a functionally symmetric population, we arbitrarily chose the right leg in the statistical analysis.

\section{Results}

\section{Improvements in objective measures of function}

Overall gait pattern deviations decreased significantly, indicated by an increase in GDI scores on the operated limb, GDI-kinetic scores on the operated and the non-operated limb (Table 2). Compared to the control group, patients with TKA demonstrated significantly lower GDI-kinetic scores and reduced walking speed after surgery, while kinematic GDI scores were comparable to the controls. Overall performance on the 5STS improved after surgery and was still significantly lower than the control group value (Table 2).

\section{Functional improvement represented by performance on the 5STS}

Patients with TKA were dichotomized into two groups based on the MDC of $2.5 \mathrm{~s}$ on the 5STS: the "Good 5STS 
Table 2 Functional assessment at baseline prior to knee arthroplasty and at 1-year follow-up in patients with knee osteoarthritis and controls

\begin{tabular}{lllll} 
TKA $(n=28)$ & & & Controls $(n=25)$ \\
$\begin{array}{llll}\text { Pre-TKA baseline } \\
\text { mean (SD) }\end{array}$ & $\begin{array}{l}\text { Mean change } \\
(\text { SD })\end{array}$ & $\begin{array}{l}\text { Effect size } \\
(95 \% \text { CI })\end{array}$ & $\begin{array}{l}\text { Post-TKA } \\
\text { mean (SD) }\end{array}$ & $\begin{array}{l}\text { Control group } \\
\text { mean (SD) }\end{array}$ \\
\hline
\end{tabular}

\begin{tabular}{|c|c|c|c|c|c|}
\hline \multicolumn{6}{|l|}{ Gait pattern } \\
\hline GDI operated & $87.2(10.5)$ & $5.8(13)^{*}$ & $0.4(0.1-0.8)$ & $93(9.9)$ & $96.6(9)$ \\
\hline GDI non-operated & $89.3(12.6)$ & $4.5(13.8)$ & $0.3(-0.1-0.7)$ & $93.9(8.7)$ & $96.6(9)$ \\
\hline GDI-kinetic operated & $90.1(6.9)$ & $3.8(6.9)^{*}$ & $0.5(0.1-0.9)$ & $93.8(8.4)$ & $100(8.6)^{\square}$ \\
\hline GDI-kinetic non-operated & $87.9(8.3)$ & $6.7(7.8)^{\frac{\hbar}{\ddagger}}$ & $0.9(0.4-1.3)$ & $94.7(8.6)$ & $100(8.6)^{\square}$ \\
\hline Walking speed $(\mathrm{m} / \mathrm{s})$ & $1.11(0.20)$ & $0.07(0.15)^{*}$ & $0.5(0.1-0.8)$ & $1.17(0.18)$ & $1.30(0.18)^{*}$ \\
\hline ND walking speed & $0.37(0.07)$ & $0.02(0.05)^{*}$ & $0.5(0.1-0.9)$ & $0.40(0.06)$ & $0.44(0.06)^{*}$ \\
\hline \multicolumn{6}{|l|}{ Self-reported function } \\
\hline \multicolumn{6}{|l|}{ KOOS $(0-100)$} \\
\hline Pain & $45.3(15.2)$ & $32.7(22)^{\ddagger}$ & $1.5(0.9-2.0)$ & $78(20.6)$ & $96.9(5.3)^{\ddagger}$ \\
\hline Symptoms & $41.2(20.5)$ & $34.6(23.1)^{\ddagger}$ & $1.5(1.0-2-0)$ & $75.9(18.3)$ & $94.9(6.7)^{\ddagger}$ \\
\hline ADL & $57.2(15.3)$ & $23.8(17.9)^{\ddagger}$ & $0.8(0.4-1.2)$ & $81.1(19.1)$ & $96.3(6.3)^{\ddagger}$ \\
\hline Sport/Rec & $22(21.8)$ & $17.1(28.3)^{\square}$ & $0.6(0.2-1.0)$ & $39.1(27)$ & $89.6(14.9)^{\ddagger}$ \\
\hline Knee-related QoL & $28.1(12)$ & $33.6(23.5)^{\ddagger}$ & $1.4(0.9-2.0)$ & $61.6(26.8)$ & $90.5(13.8)^{\ddagger}$ \\
\hline \multicolumn{6}{|l|}{ EQ-5D } \\
\hline Score $(-0.594-1)$ & $0.61(0.22)$ & $0.14(0.27)^{\square}$ & $0.5(0.1-0.9)$ & $0.75(0.26)$ & $0.93(0.1)^{\ddagger}$ \\
\hline \multicolumn{6}{|l|}{ Performance-based function } \\
\hline 5STS, s & $14.5(4.2)$ & $-1.6(4.4)^{\square}$ & $0.6(0.2-1.0)$ & $12.8(4.8)$ & $9.9(2.9)^{\square}$ \\
\hline
\end{tabular}

$T K A$ total knee arthroplasty; $S D$ standard deviation; $C I$ confidence interval; $n$ number; $G D I$ Gait Deviation Index; $N D$ non-dimensionalized; KOOS Knee Injury and Osteoarthritis Outcome Score; $A D L$ activities of daily living; Sport/Rec sport and recreation; $Q o L$ quality of life, $E Q-5 D$ EuroQol 5 Dimensions; 5STS Five Times Sit-to-Stand. Parametric and nonparametric statistics were used; paired samples $t$ test and Wilcoxon Sign rank test to compare pre- and post-operative results within the TKA group; independent samples $t$ test and Mann Whitney $U$ test to calculate differences between TKA group post-operatively and controls, which are reported in the column for control group mean. Level of significance set to $* p<0.05, \square p<0.01,{ }^{\ddagger} p<0.001$

outcome" ( $n=10,36 \%)$ and the "Bad 5STS outcome" group $(n=18,64 \%)$. Significant improvements were found in GDI and GDI-kinetic scores in the "Good 5STS outcome" group (Fig. 2). The "Good 5STS outcome" group demonstrated improvements in GDI scores on the operated limb and GDI-kinetic scores on both the operated and nonoperated limb, respectively. In the "Bad 5STS outcome" group, no significant changes were found in GDI scores, while GDI-kinetic scores on non-operated limb increased significantly (Fig. 2).

\section{Improvements in self-reported measures of function and pain}

Data from the KOOS and EQ-5D indicated improvement in all areas, pain, symptoms, function in ADL, function in sport and recreation, knee-related quality of life, and HRQoL (Table 2). The largest functional improvements were found in KOOS subscales "pain" (73\%), "symptoms"

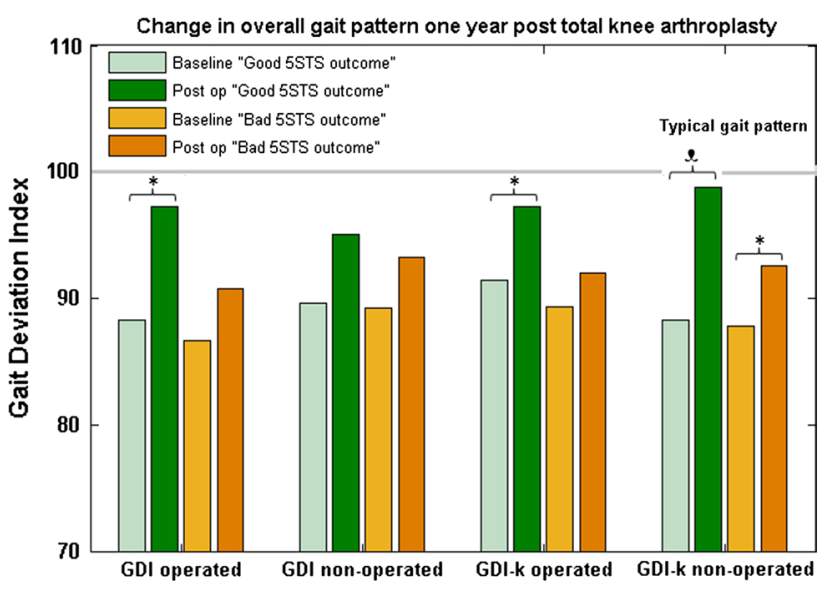

Fig. 2 Overall gait pattern, quantified using the Gait Deviation Index for kinematics (GDI) and kinetics (GDI-k), at baseline and 1 year after total knee arthroplasty in patients with knee osteoarthritis. Patients were grouped by change in performance on the Five Times Sit-to-Stand test. Level of significance set to $* p<0.05, \square p<0.01$ 


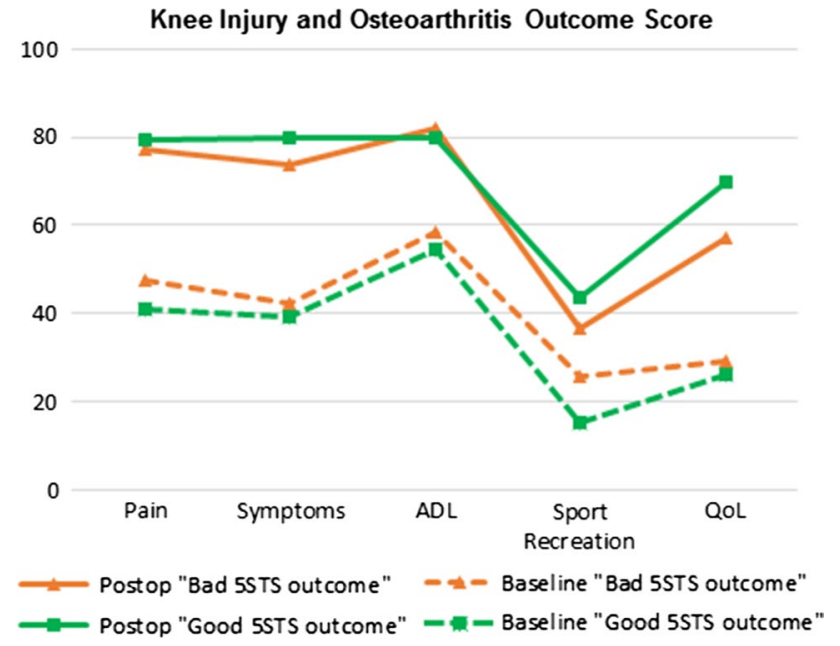

Fig. 3 Patient-reported function, evaluated using the Knee Injury and Osteoarthritis Outcome Score (KOOS), at baseline and 1 year after total knee arthroplasty in patients with knee osteoarthritis. Patients were grouped by change in performance on the Five Times Sit-toStand test. ADL, activities of daily living; QoL, knee-related quality of life

$(85 \%)$ and "knee-related quality of life" (121\%). Compared to controls, lower scores were found in all subscales of KOOS and in EQ-5D (Table 2). When comparing KOOS subscale scores between the "Good" and "Bad" 5STS outcome groups among patients with TKA, no significant differences were found in either subscale (Fig. 3).

\section{Discussion}

The most important finding of the present study was that objectively measured function, represented by gait patterns and the 5STS, improved significantly, but did not reach the level of healthy controls. Based on change in 5STS performance, we identified patients with substantial improvements in gait patterns, while self-reported measures of function could not differentiate between patients improving in functional performance and those who did not.

Pre-operatively, lower GDI-kinetic scores were observed in the contralateral limb compared to the soon-to-be-operated limb. After surgery, the largest gait pattern improvements were found in GDI-kinetic scores of the contralateral (non-operated) limb. These results suggest TKA has a positive impact on gait patterns for both limbs, although deviations are still present 1 year post-operatively as compared to healthy controls. These results corroborate the findings of Metcalfe et al. [23] that gait pattern recovery at the contralateral knee is variable and often incomplete. Using a 1-year follow-up of gait patterns may be a too short period of time with regard to restoring joint loading. It has been suggested that functional recovery continues beyond 1 year and even up to 2 years after surgery [20].

We hypothesized that performance on the 5STS would improve after surgery, and in this sample the performance improved significantly. Nevertheless, the improvement only exceeded the MDC in 10 (36\%) of the 28 TKA patients, while the rest remained unchanged. Patients classified as having a "Good 5STS outcome" displayed significant improvements in GDI and GDI-kinetic scores, whereas patients classified as having a "Bad 5STS outcome" did not. Performance on this test is associated with quadriceps strength, and this test is considered a surrogate measure for lower limb strength [22, 27, 31]. Studies also report factors such as balance, age, weight, and sensorimotor competence play an important role in 5STS performance [22, 31]. Christiansen et al. [6] evaluated weight-bearing asymmetry during the 5STS in patients with knee osteoarthritis and concluded that greater amounts of weight-bearing asymmetry correlated with poorer functional performance up to 6 months after TKA. Alnahdi et al. [1] found patients with TKA displayed unloading of the operated limb, shifting the load to the contralateral limb when performing a sit-tostand test 1 year after surgery.

Comparing self-reported function and pain between the "Good 5STS outcome" and "Bad 5STS outcome" groups, we found no differences, suggesting that self-reported measures are not able to detect change in performance on this test. Results of our study are consistent with prior studies demonstrating that improvements in self-reported measures are greater than improvement in performancebased function [17, 25, 35]. Boonstra et al. [3] suggest self-reported measures of function are highly influenced by pain. After a successful TKA, pain is substantially reduced and this pain relief may lead patients to overestimate selfreported function or confuse improvements in function with improvements in pain [3].

Limitations of the present study include that our sample consisted of individuals without comorbidities who were able to ambulate without the use of a walking aid. Consequently, we cannot generalize the results to all patients with knee osteoarthritis. The sample size is small, yet consistent with, and even larger than other studies using 3D gait analysis $[18,23,36]$. However, these patients presented with significantly higher weight and BMI compared to the control group, and this difference in BMI may have influenced gait patterns and 5STS performance [31]. We recognize that the use of seven different senior consultant orthopaedic surgeons to perform the surgeries increases the generalizability of the results, as this is representative of surgical practice. However, individual capability of an orthopaedic surgeon could be argued to influence the outcome of the surgery. Examining the inter-surgeon effect was beyond the scope of the present study. Additionally, there was no standardization of the 
post-operative rehabilitation following TKA to control the impact of rehabilitation on gait pattern or 5STS performance. Anecdotally, the standard protocol for rehabilitation following TKA did not differ between the two orthopaedic departments in this study. The rehabilitation programme included inpatient physiotherapy ( $>1$ week), followed by physiotherapy in a primary care setting of the patient's choice for varying lengths of time. Median time for post-operative rehabilitation in this sample was 3 months. The MDC of the 5STS is based on a sample of 29 females, mean aged 73.6 years who were able to walk at least $10 \mathrm{~m}$ and stand at least $10 \mathrm{~min}$ without an assistive device and who had no neurological disease, diabetes, visual deficits, or amputated extremities [14]. Caution should be taken not to overestimate the importance of this value, since the two studied groups (ours and Goldberg's) might differ substantially. Additionally, we used the MDC reported for GDI [10], to evaluate change in GDIkinetic scores. Since the GDI-kinetic is an analogue of the GDI and the magnitude of change of the two measures was similar, the MDC of 5.4 units was considered to be useful in the evaluation of GDI-kinetic.

Based on our findings, we advocate for use of the 5STS in clinical practice, as improvement beyond the MDC in 5STS performance appears to be accompanied by significant reductions in kinematic and kinetic gait pattern deviations. Additionally, our data indicate self-reported measures of function have limited use in detecting change in performance-based function. This has implications for pre-operative patient education. Specifically, we believe it is important to inform patients undergoing surgery that TKA is an effective treatment for alleviating pain and symptoms of the affected joint, but activities of daily living and more strenuous activities requiring power, strength, and balance may not become fully restored after surgery. Future research should evaluate the impact of specific exercise programmes on restoring normal joint loading patterns and functional performance. Additionally, we recommend studies to examine whether more extensive post-operative rehabilitation could lead to greater gains in performance-based function and in reductions in kinetic gait deviations. It would also be of interest to evaluate whether joint loading deviations persist at two or more years after surgery.

\section{Conclusion}

Measures of overall gait patterns and the 5STS revealed improvements in function 1 year after TKA, but were not restored to the level of healthy controls. Based on change in 5STS performance, we identified patients with substantial improvements in gait patterns. Self-reported measures of function could not detect differences between patients improving in 5STS performance and those who did not. These findings highlight the use of the 5STS in clinical practice since improvement on this test seems to follow the reduction in gait pattern deviations.

Acknowledgments We would like to acknowledge senior orthopaedic surgeon Per Gedin and data technician Mikael Reimeringer for contributing to the data collection. This study was supported by grants from Karolinska Institutet, Promobilia, the Swedish Rheumatism Foundation, and the Developmental foundation of the footwear industry. Study sponsors had no involvement in study design, collection, analysis and interpretation of data; in the writing of the manuscript; or in the decision to submit the manuscript for publication.

Author contributions JEN, MI, EWB involved in conception and design, drafting the article. JEN, ACE, MH contributed to acquisition of data. All authors have made substantial contributions in the interpretation of data, revising the article critically, and all approved of the final version for submission.

\section{Compliance with ethical standards}

Conflict of interests None to declare.

Open Access This article is distributed under the terms of the Creative Commons Attribution 4.0 International License (http://creativecommons.org/licenses/by/4.0/), which permits unrestricted use, distribution, and reproduction in any medium, provided you give appropriate credit to the original author(s) and the source, provide a link to the Creative Commons license, and indicate if changes were made.

\section{References}

1. Alnahdi AH, Zeni JA, Snyder-Mackler L (2015) Quadriceps strength asymmetry predicts loading asymmetry during sit-to-stand task in patients with unilateral total knee arthroplasty. Knee Surg Sports Traumatol Arthrosc. doi:10.1007/ s00167-015-3827-x

2. Becker R, Doring C, Denecke A, Brosz M (2011) Expectation, satisfaction and clinical outcome of patients after total knee arthroplasty. Knee Surg Sports Traumatol Arthrosc 19(9):1433-1441

3. Boonstra MC, De Waal Malefijt MC, Verdonschot N (2008) How to quantify knee function after total knee arthroplasty? Knee 15(5):390-395

4. Brooks RG, Jendteg S, Lindgren B, Persson U, Bjork S (1991) EuroQol: health-related quality of life measurement. Results of the Swedish questionnaire exercise. Health Policy 18(1):37-48

5. Campbell MJ (2007) Medical statistics: a textbook for the health sciences, 4th edn. Wiley, Chichester

6. Christiansen CL, Stevens-Lapsley JE (2010) Weight-bearing asymmetry in relation to measures of impairment and functional mobility for people with knee osteoarthritis. Arch Phys Med Rehabil 91(10):1524-1528

7. Collins NJ, Prinsen CA, Christensen R, Bartels EM, Terwee CB, Roos EM (2016) Knee Injury and Osteoarthritis Outcome Score (KOOS): systematic review and meta-analysis of measurement properties. Osteoarthr Cartil / OARS, Osteoarthr Res Soc. doi:10.1016/j.joca.2016.03.010

8. Davis R, Ounpuu S, Tybursk D, Gage J (1991) A gait analysis data collection and reduction technique. Hum Mov Sci 10(5):575 
9. Dieppe P, Judge A, Williams S, Ikwueke I, Guenther KP, Floeren M, Huber J, Ingvarsson T, Learmonth I, Lohmander LS, Nilsdotter A, Puhl W, Rowley D, Thieler R, Dreinhoefer K (2009) Variations in the pre-operative status of patients coming to primary hip replacement for osteoarthritis in European orthopaedic centres. BMC Musculoskelet Disord 10:19

10. Dolan P, Roberts J (2002) Modelling valuations for Eq-5d health states: an alternative model using differences in valuations. Med Care 40(5):442-446

11. Esbjornsson AC, Rozumalski A, Iversen MD, Schwartz MH, Wretenberg P, Brostrom EW (2014) Quantifying gait deviations in individuals with rheumatoid arthritis using the Gait Deviation Index. Scand J Rheumatol 43(2):124-131

12. Eve L, Mc Nee A, Shortland A (2006) Extrinsic and intrinsic variation in kinematic data from the gait of healthy adult subjects. Gait Posture 24(Supplement 2):S56-S57

13. Ferrari A, Benedetti MG, Pavan E, Frigo C, Bettinelli D, Rabuffetti M, Crenna P, Leardini A (2008) Quantitative comparison of five current protocols in gait analysis. Gait Posture 28(2):207-216

14. Goldberg A, Chavis M, Watkins J, Wilson T (2012) The fivetimes-sit-to-stand test: validity, reliability and detectable change in older females. Aging Clin Exp Res 24(4):339-344

15. Group KATT, Johnston L, MacLennan G, McCormack K, Ramsay C, Walker A (2009) The Knee Arthroplasty Trial (KAT) design features, baseline characteristics, and two-year functional outcomes after alternative approaches to knee replacement. J Bone Joint Surg Am 91(1):134-141

16. Hof AL (1996) Scaling gait data to body size. Gait Posture 4(3):222-223

17. Hossain FS, Konan S, Patel S, Rodriguez-Merchan EC, Haddad FS (2015) The assessment of outcome after total knee arthroplasty: are we there yet? Bone Joint J 97-B(1):3-9

18. Joglekar S, Gioe TJ, Yoon P, Schwartz MH (2012) Gait analysis comparison of cruciate retaining and substituting TKA following PCL sacrifice. Knee 19(4):279-285

19. Jones CA, Voaklander DC, Johnston DW, Suarez-Almazor ME (2000) Health related quality of life outcomes after total hip and knee arthroplasties in a community based population. J Rheumatol 27(7):1745-1752

20. Kleijn LL, van Hemert WL, Meijers WG, Kester AD, Lisowski L, Grimm B, Heyligers IC (2007) Functional improvement after unicompartmental knee replacement: a follow-up study with a performance based knee test. Knee Surg Sports Traumatol Arthrosc 15(10):1187-1193

21. Komnik I, Weiss S, Fantini Pagani CH, Potthast W (2015) Motion analysis of patients after knee arthroplasty during activities of daily living-a systematic review. Gait Posture 41(2):370-377

22. Lord SR, Murray SM, Chapman K, Munro B, Tiedemann A (2002) Sit-to-stand performance depends on sensation, speed, balance, and psychological status in addition to strength in older people. J Gerontol A Biol Sci Med Sci 57(8):M539-543

23. Metcalfe A, Stewart C, Postans N, Barlow D, Dodds A, Holt C, Whatling G, Roberts A (2013) Abnormal loading of the major joints in knee osteoarthritis and the response to knee replacement. Gait Posture 37(1):32-36
24. Milner CE (2009) Is gait normal after total knee arthroplasty? Systematic review of the literature. J Orthop Sci 14(1):114-120

25. Mizner RL, Petterson SC, Clements KE, Zeni JA Jr, Irrgang JJ, Snyder-Mackler L (2011) Measuring functional improvement after total knee arthroplasty requires both performance-based and patient-report assessments: a longitudinal analysis of outcomes. J Arthroplasty 26(5):728-737

26. Nakagawa S, Cuthill IC (2007) Effect size, confidence interval and statistical significance: a practical guide for biologists. Biol Rev Camb Philos Soc 82(4):591-605

27. Newcomer KL, Krug HE, Mahowald ML (1993) Validity and reliability of the timed-stands test for patients with rheumatoid arthritis and other chronic diseases. J Rheumatol 20(1):21-27

28. Obradovic M, Lal A, Liedgens H (2013) Validity and responsiveness of EuroQol-5 dimension (EQ-5D) versus Short Form-6 dimension (SF-6D) questionnaire in chronic pain. Health Qual Life Outcomes 11:110

29. Roos E, Roos H, Lohmander L, Ekdahl C, Beynnon B (1998) Knee Injury and Osteoarthritis Outcome Score (KOOS)-development of a self-administered outcome measure. J Orthop Sports Phys Ther 28(2):88-96

30. Rozumalski A, Schwartz MH (2011) The GDI-Kinetic: a new index for quantifying kinetic deviations from normal gait. Gait Posture 33(4):730-732

31. Schenkman M, Hughes MA, Samsa G, Studenski S (1996) The relative importance of strength and balance in chair rise by functionally impaired older individuals. J Am Geriatr Soc 44(12):1441-1446

32. Schwartz MH, Rozumalski A (2008) The Gait Deviation Index: a new comprehensive index of gait pathology. Gait Posture 28(3):351-357

33. Skou ST, Roos EM, Laursen MB, Rathleff MS, Arendt-Nielsen L, Simonsen O, Rasmussen S (2015) A randomized, controlled trial of total knee replacement. N Engl J Med 373(17):1597-1606

34. Steinhoff AK, Bugbee WD (2014) Knee Injury and Osteoarthritis Outcome Score has higher responsiveness and lower ceiling effect than Knee Society Function Score after total knee arthroplasty. Knee Surg Sports Traumatol Arthrosc. doi:10.1007/ s00167-014-3433-3

35. Stevens-Lapsley JE, Schenkman ML, Dayton MR (2011) Comparison of self-reported knee injury and osteoarthritis outcome score to performance measures in patients after total knee arthroplasty. PMR 3(6):541-549; quiz 549

36. van den Boom LG, Halbertsma JP, van Raaij JJ, Brouwer RW, Bulstra SK, van den Akker-Scheek I (2014) No difference in gait between posterior cruciate retention and the posterior stabilized design after total knee arthroplasty. Knee Surg Sports Traumatol Arthrose 22(12):3135-3141

37. Wiik AV, Aqil A, Tankard S, Amis AA, Cobb JP (2015) Downhill walking gait pattern discriminates between types of knee arthroplasty: improved physiological knee functionality in UKA versus TKA. Knee Surg Sports Traumatol Arthrosc 23(6):1748-1755

38. Wylde V, Dieppe P, Hewlett S, Learmonth ID (2007) Total knee replacement: is it really an effective procedure for all? Knee 14(6):417-423 